University of Wollongong

Research Online

Australian Institute for Innovative Materials -

Papers

Australian Institute for Innovative Materials

$1-1-2009$

\title{
Microwave absorption measurements using broad-band meanderline approach
}

Ching-Ching Tsai

Chang Jung University, Taiwan

J Choi

University of Ulsan, Republic of Korea

Sunglae Cho

University of Ulsan, Republic of Korea

S J. Lee

Hanyang University, Republic of Korea

Bimal K. Sarma

University of Wisconsin, USA

See next page for additional authors

Follow this and additional works at: https://ro.uow.edu.au/aiimpapers

Part of the Engineering Commons, and the Physical Sciences and Mathematics Commons

Research Online is the open access institutional repository for the University of Wollongong. For further information contact the UOW Library: research-pubs@uow.edu.au 


\title{
Microwave absorption measurements using broad-band meanderline approach
}

\author{
Abstract \\ We describe a technique that permits broad-band, field-dependent ferromagnetic and electron \\ paramagnetic resonance absorption measurements that is applicable to thin films and patterned micro- \\ /nanostructured arrays and is based on a wire-wound meanderline approach. Techniques to prepare \\ meanderlines and perform microwave measurements are described along with some demonstrations \\ involving an electron paramagnetic resonance calibration/test material, 2,2-diphenyl-1-picryl-hydrazyl, and \\ a ferromagnetic cobalt thin film. \\ Keywords \\ Microwave, absorption, measurements, using, broad, band, meanderline, approach \\ Disciplines \\ Engineering | Physical Sciences and Mathematics \\ Publication Details \\ Tsai, C, Choi, J, Cho, S, Lee, SJ, Sarma, BK, Thompson, C, Chernyashevskyy, O, Nevirkovets, I \& Ketterson, \\ JB (2009), Microwave absorption measurements using broad-band meanderline approach, Review of \\ Scientific Instruments, 80(2), pp. 023904-1-023904-8.

\section{Authors} \\ Ching-Ching Tsai, J Choi, Sunglae Cho, S J. Lee, Bimal K. Sarma, C Thompson, O Chernyashevskyy, Ivan \\ Nevirkovets, and J B. Ketterson
}




\title{
Microwave absorption measurements using a broad-band meanderline approach
}

\author{
C. C. Tsai, ${ }^{1}$ J. Choi, ${ }^{2}$ Sunglae Cho, ${ }^{2}$ S. J. Lee, ${ }^{3}$ B. K. Sarma, ${ }^{4}$ C. Thompson, ${ }^{5}$ \\ O. Chernyashevskyy, ${ }^{5}$ I. Nevirkovets, ${ }^{5}$ and J. B. Ketterson ${ }^{5,6}$ \\ ${ }^{1}$ Department of Engineering and Management of Advanced Technology, Chang Jung Christian University, \\ Tainan 71101, Taiwan \\ ${ }^{2}$ Department of Physics, University of Ulsan, Ulsan 680-749, Republic of Korea \\ ${ }^{3}$ Department of Physics, Hanyang University, Seoul 133-791, Republic of Korea \\ ${ }^{4}$ Department of Physics, University of Wisconsin, Milwaukee, Wisconsin 53201, USA \\ ${ }^{5}$ Department of Physics, Northwestern University, Evanston, Illinois 60208, USA \\ ${ }^{6}$ Department of Electrical and Computer Engineering, Northwestern University, Evanston, \\ Illinois 60208, USA
}

(Received 17 August 2008; accepted 21 December 2008; published online 11 February 2009)

\begin{abstract}
We describe a technique that permits broad-band, field-dependent ferromagnetic and electron paramagnetic resonance absorption measurements that is applicable to thin films and patterned micro-/nanostructured arrays and is based on a wire-wound meanderline approach. Techniques to prepare meanderlines and perform microwave measurements are described along with some demonstrations involving an electron paramagnetic resonance calibration/test material, 2,2-diphenyl-1-picryl-hydrazyl, and a ferromagnetic cobalt thin film. (c) 2009 American Institute of Physics. [DOI: 10.1063/1.3070471]
\end{abstract}

\section{INTRODUCTION}

As a result of recent advances in nanometer fabrication techniques, coupled with the industry-wide drive to increase information density in magnetic recording, there is a growing interest, both fundamental and technological, in patterned nanometer scale magnetic thin films. By virtue of their extremely small size, these nanometer scale structures possess unique properties that differ from their parent bulk material(s), ${ }^{1-4}$ which make them potential candidates for various advanced magnetoelectronic devices. ${ }^{5-8}$ Further device development may well depend on a better understanding of various dynamic magnetic phenomena in such nanostructures.

It is well known that ferromagnetic resonance (FMR) is a powerful experimental tool for probing the fundamental magnetic properties in magnetically ordered materials. Recently a method was developed to numerically calculate the absorption spectra associated with various modes excited by a position- and time-dependent rf field in the presence of a static field as well as the internal exchange and anisotropy fields in a periodic lattice of nanoparticles. ${ }^{3}$ Experimental observation of the spectra would further corroborate the applicability of the underlying model and the numerical techniques employed in the calculations. However, to better understand the magnetization dynamics of the ferromagnetic state of the patterned thin films, particularly in various nonlinear regimes, it is highly desirable to utilize a broad-band FMR spectrometer.

The techniques typically employed tend to involve either a narrow band resonator or broader-band transmission approaches. Narrow band approaches have the virtue of high sensitivity and traditionally employ a microwave cavity. When studying FMR on films and patterned nanostructures, cavities are often replaced by resonant or nonresonant strip lines or loops; many useful properties of individual submicron structures can be measured in this way. In this article, we describe in detail techniques for broad-band FMR studies on thin films and nanostructured arrays that are patterned over millimeter scales using a transmission methodology.

\section{APPARATUS}

Our FMR spectrometer was designed to operate in a transmission mode. One of the key features is the design of the probe utilized to measure the electromagnetic response from the sample. Motivated by the patterned microstrip configurations employed in some FMR measurements, ${ }^{9-15}$ we utilize a meanderline. Figure 1 illustrates schematically an outline of the device, which generates rf magnetic fields that are both in plane and out of plane with respect to the sample.

We have not performed a simulation of the magnetic field distribution associated with our meanderlines; in practice it will depend on various geometrical factors and the nature of the sample loading it, especially in the high frequency regime. However, qualitatively it is clear that the magnetic fields in the region midway between each wire of the meanderlines lie out of plane, whereas the magnetic fields directly under the wire (adjacent to the sample) will be largely in plane.

Two methods were developed to fabricate meanderlines. Both methods were found to produce well formed lines with both a controlled spacing between turns and precise overall dimensions. The first method was relatively straightforward. A platform (Fig. 2) was constructed to facilitate meanderline fabrication. The setup contained two square brass bars $(0.1$ $\times 0.1 \times 1.0 \mathrm{in}^{3}$ ) that formed a kind of loom. Each was machined to form 20 parallel grooves [Fig. 2(b)]. The two bars, 


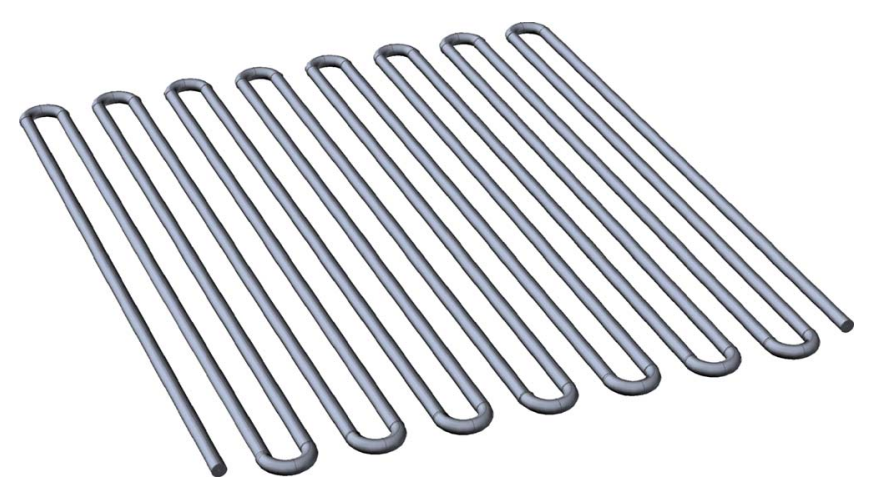

FIG. 1. (Color online) A schematic of the meanderline.

which were fixed to the platform (either with glue or screws entering from the bottom), were aligned parallel to each other with the distance between them set to conform with the nominal overall width dimension chosen for the line. The bars were positioned in such a way that successive grooves on opposing bars were perpendicular to each other (thereby ensuring that the wires will be perpendicular to the bars). Two sets of bars having slightly different groove sizes (one with width of $0.010 \mathrm{in}$. and depth of $0.010 \mathrm{in}$. and the other with width of 0.013 in. and depth of 0.013 in.) were machined in order to accommodate two different wire sizes (American Wire Gauges 30 and 28, respectively) used here for making the meanderlines. S00311 dry film Poly(tetrafluoroethylene) mold release antistick agent from Diversified Brands was presprayed on the surface of the grooves and the surrounding area. The wire was then wound in a zigzag fashion while being anchored into each of the grooves of the loom; as the winding progressed, a slab [Fig. 2(a)] was advanced over the filled grooves so as to anchor each turn firmly in place. A certain tension is required to assure that the wire remains straight and does not loosen. However, care must be taken not to apply too much tension, as the sharp edges of the grooves may sever the wire. After the desired number of turns is completed with the wire positioned in the grooves, varnish (GE 7031) or epoxy adhesive was applied

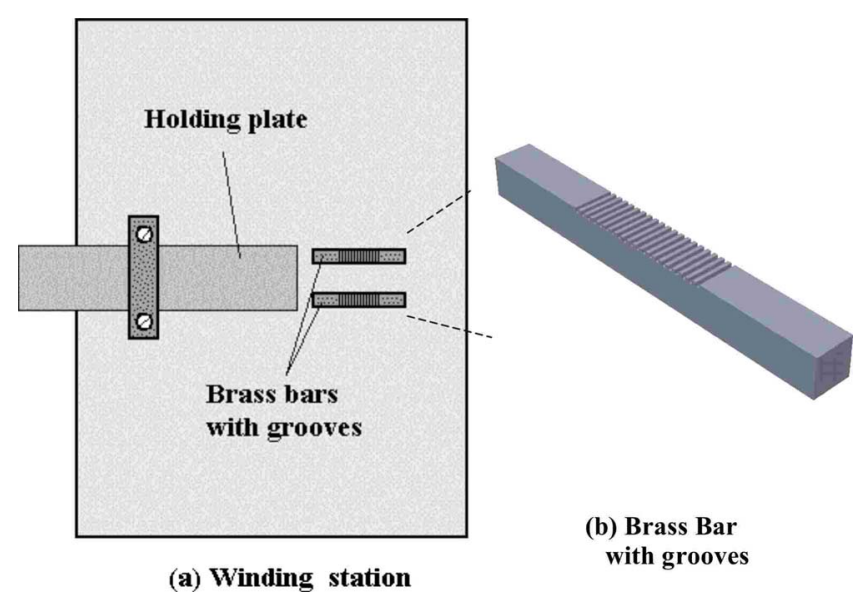

FIG. 2. (Color online) A "winding station" to facilitate meanderline fabrication. (a) A drawing of the station. (b) One of the grooved brass bars.

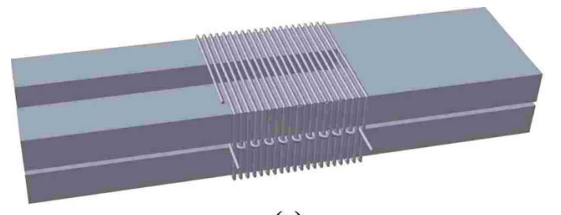

(a)

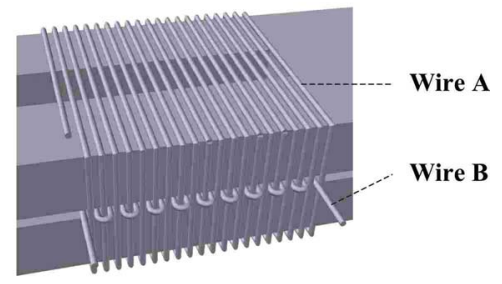

(b)

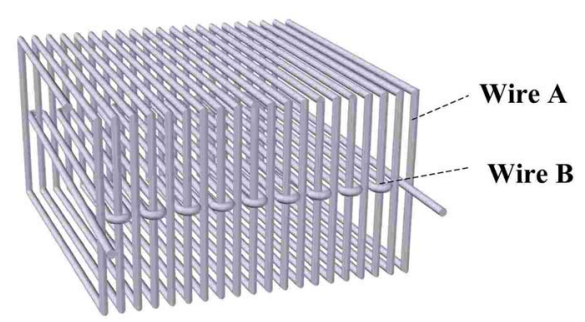

(c)

FIG. 3. (Color online) (a) A drawing of a facility for meanderline fabrication. (b) Close-up view showing the interlacing between wires A and B. (c) A clearer view of wire A and wire B with metal slabs transparent.

so that the line will subsequently retain its structure. Once the varnish or epoxy has set, the meanderline is then ready to be transferred to the probe.

We now describe an alternative method for making meanderlines that allows independent control of the turn spacing. A winding facility was constructed involving two rectangular slabs of metal, with one on top of the other but separated by a distance slightly larger than the diameter of the wire used for the meanderline (Fig. 3). A rectangular slot was cut from one of the slabs to allow the later insertion of epoxy. Antistick agent was sprayed over all surfaces of the two metal slabs.

The strategy used here was to interlace (weave) two separate lengths of wire (wires A and B in Fig. 3) within each other, using one of the wires (wire $\mathrm{A}$ in the figure) as a spacer for the other wire (wire B) that forms the meanderline. Wire A was coiled around the two metal slabs while wire B was entwined within the space between the two metal slabs. After the winding is completed, some epoxy was applied to wire B (through the slot mentioned above) to fix the structure before transferring it to the probe.

Figure 4 shows some of the meanderlines constructed by the methods described here. The first row shows three different configurations of the meanderlines fabricated from gauge 30 (diameter of 0.010 in.) copper wire. The second row shows another three configurations made from a different size of copper wire (gauge 28, diameter of 0.013 in.). Some meanderlines were also made from gauge 28 niobium wire for low temperature applications where it becomes superconducting. The configuration of the meanderlines is one of the 


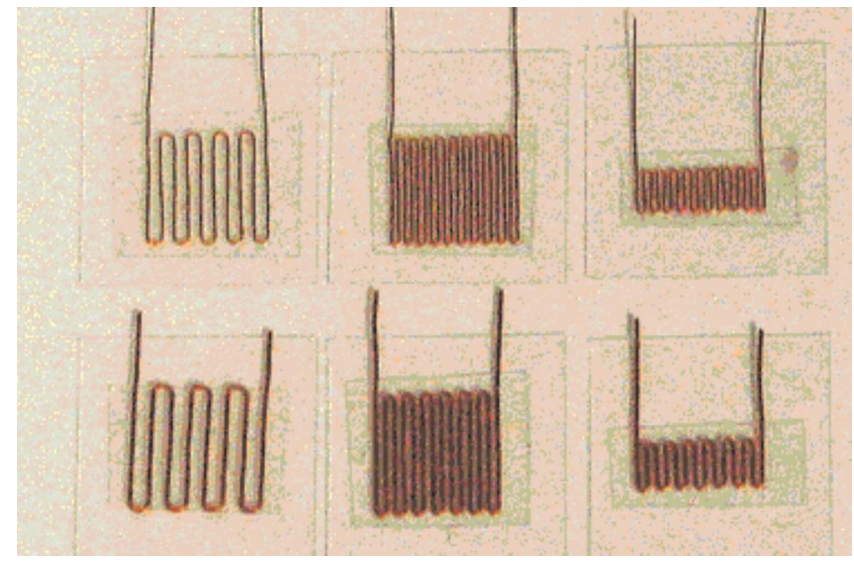

FIG. 4. (Color online) Some fabricated meanderlines (before application of varnish).

factors that determine the electromagnetic transmission characteristics of the system. (Some measured electromagnetic transmission characteristics will be discussed below.)

Another important feature of the spectrometer is the ability to in situ rotate the sample within the probe. This feature allows the experimenter to study the response of the sample to different orientations of the dc magnetic field while minimizing any changes in the coupling that might otherwise occur within the housing of the meanderline on removing and reorienting the sample.

We utilized two designs, which are shown in Figs. 5 and 6 , for housing the meanderline and the sample. The housing was made from copper and the assembly has lateral dimensions of $1 \times 1 \mathrm{in}^{2}$. Microwaves are coupled into and out of the housing through two SMA connectors [not shown but positioned on top of the copper tubes in Figs. 5(a) and 6(a)]. The meanderline was mounted on the top plate $[\mathrm{a} 1 \times 1.5$ $\times 0.2$ in $^{3}$ plate in Fig. 5(b) and a 1 in. diameter, 0.1 in. thick

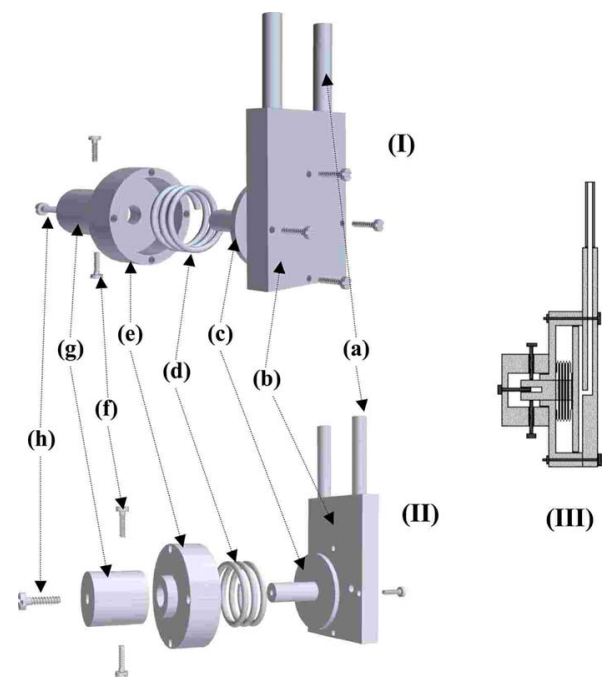

FIG. 5. (Color online) A side mounting housing assembly for holding the meanderline and the sample. [(I) and (II)] Side views of the assembly. (III) Cross-sectional diagram of the assembly. (a) Copper tubes for the input and output transmission lines; (b) top plate for mounting the meanderline; (c) sample holder; (d) compression springs; (e) receptacle cup; (f) set screws for holding the sample holder from rotation; (g) rotator; (h) screw for sample holder and rotator coupling.
(I)

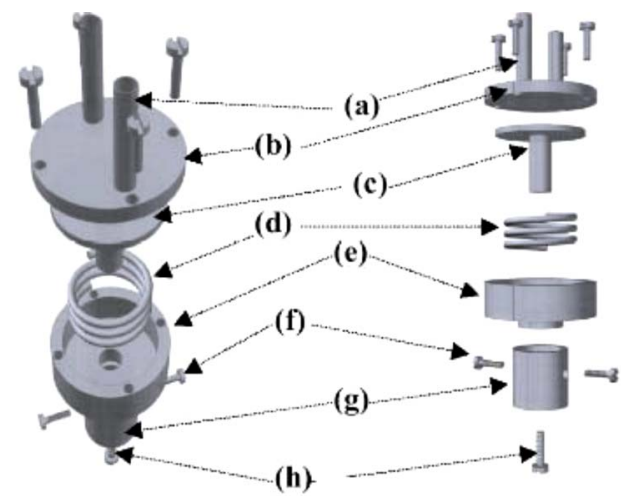

(II)

\section{(III)}
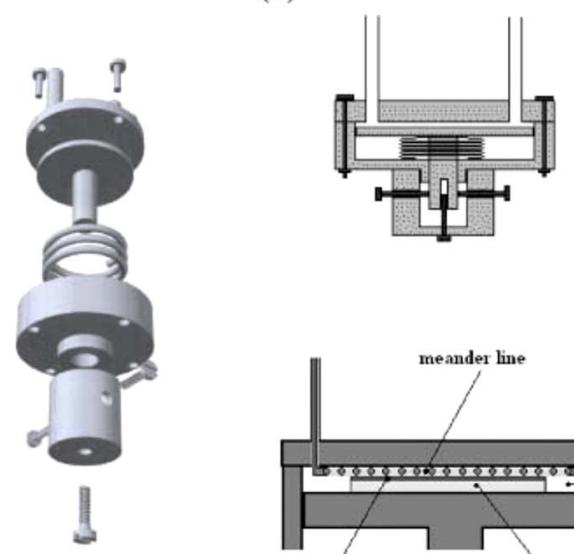

(IV)

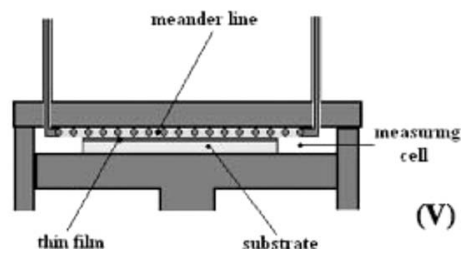

FIG. 6. (Color online) An axisymmetric housing assembly for the meanderline and the sample. (I) Top view. (II) Side view. (III) Bottom view. (IV) Cross-sectional diagram of the assembly. (v) Positioning of the sample within the sample holder. (a) Copper tubes for input and output transmission lines; (b) top plate for mounting the meanderline; (c) sample holder; (d) compression springs; (e) receptacle cup; (f) set screws for holding the sample holder from rotation; (g) rotator; (h) screw for sample holder and rotator coupling.

disk in Fig. 6(b)] and connected to the transmission lines through the two copper tubes shown in Figs. 5(a) and 6(a). The sample was glued onto the center of a swivel sample holder [Figs. 5(c) and 6(c)] and placed face down against the top of the meanderline.

A space [Figs. 5(e) and 6(d)] was machined within the top plate to contain the swivel and a loading spring (see below) and this assembly, together with the meanderline and the sample, was screwed to the plate. The sample holder was fastened to a rotation shaft [the rotator, Figs. 5(g) and 6(g)] with a screw [Figs. 5(h) and 6(h)]. This screw was also used to adjust the space within the cell housing, i.e., the gap between the top plate and the sample holder that in turn fixes the available space between the meanderline and the sample. This feature allows us to install samples having various thicknesses into the space of the measuring cell. The sample holder and the rotation shaft were held together by two set screws [Figs. 5(f) and 6(f)]. A compression spring made of phosphor bronze [Figs. 5(d) and 6(d)] was placed between the sample holder and the receptacle. The spring ensures that the sample properly contacts the meanderline and also prevents the sample surface from being damaged when rotating it by the following disengagement procedure: By pulling back on the rotation shaft we can disengage the sample from 


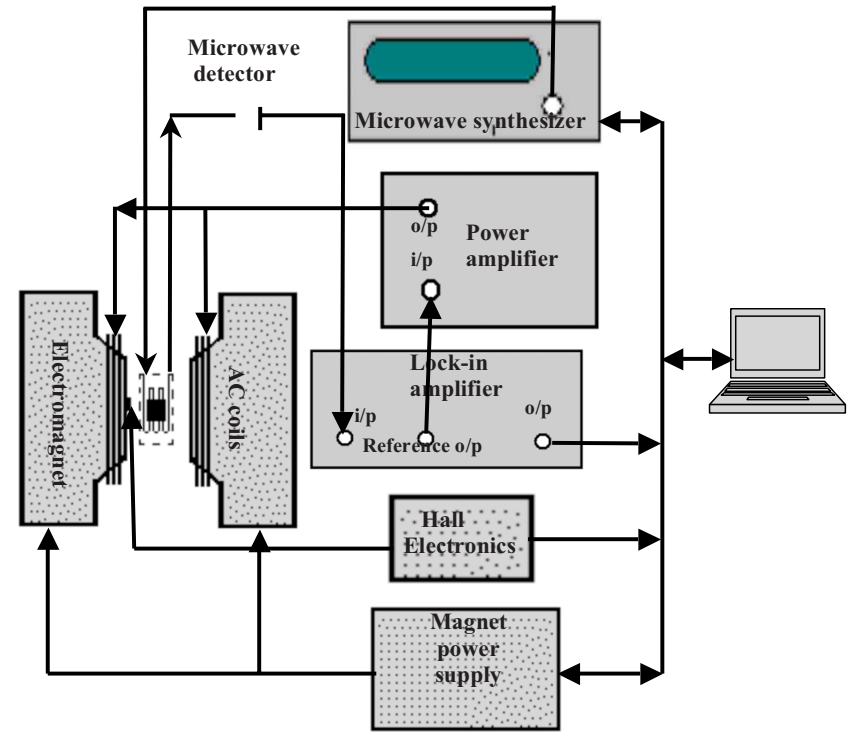

FIG. 7. (Color online) Electronics setup for the FMR spectrometer: The HP83623A microwave synthesizer generates either a cw, an AM, or a FM microwave signal in addition to permitting frequency sweeps. The output signal from the meanderline goes through the microwave diode detector and is then applied to the input of the lock-in. The Varian electromagnet was energized by the accompanying power supply, and the resulting magnetic field was monitored by the Hall electronics. The ac output from the lock-in is applied to the power amplifier that drives the field coils, thereby generating an ac modulation field. The computer recorded the detected signal output of the lock-in along with the output from the Hall probe. The field was swept by a ramp generated either by the computer or a function generator that was applied to the magnet power supply.

the meanderline; the sample can then be safely turned to some desired angle. Releasing the rotation shaft allows the sample to again contact the meanderline.

The block diagram of the electronic setup used is illustrated in Fig. 7. The microwave synthesizer utilized here was a model HP83623A manufactured by Hewlett Packard. Although it was primarily operated in the cw mode, the instrument allows frequency sweeping, $\mathrm{AM}$ and $\mathrm{FM}$, and has a leveled power output in the range from -20 to $+25 \mathrm{dBm}$ for frequencies between $10 \mathrm{MHz}$ and $20 \mathrm{GHz}$. Microwaves were transmitted from the synthesizer through a semirigid $\mathrm{Cu}$ $50 \Omega$ coaxial transmission line and coupled to the meanderline through a SMA connector. We chose coax instead of waveguide for our microwave transmission line as it simplifies coupling and allows operation over a broad range of frequencies. The output signal from the meanderline then goes to a $(0-18 \mathrm{GHz})$ microwave diode detector, the output of which was applied to the input of a Princeton HR-8 lock-in detector. For some measurements a microwave amplifier was inserted ahead of the diode.

The assembled cell, housing the meanderline and the sample, was placed between the pole pieces of a Varian electromagnet equipped with a turntable that allows the magnet to rotate through $360^{\circ}$. The gap between the magnet pole pieces was also adjustable; the maximum field was $8500 \mathrm{G}$ for a $1 \mathrm{in}$. gap at a current of $80 \mathrm{~A}$, which was supplied by a Varian V2901 regulated magnet power supply. The intensity of the magnetic field was monitored by a HGT-1010 transverse-type Hall probe chip from Lakeshore, positioned in the center of the magnet pole pieces. To perform field modulation measurements, an ac, typically $200 \mathrm{~Hz}$, from a $20 \mathrm{~W}$ power amplifier was applied to a pair of homemade Helmholtz coils that surrounded and were coaxial with the magnet pole pieces, and which in conjunction with the power amplifier provided fields of up to $40 \mathrm{G}$. The input of the power amplifier was obtained from the reference output of the lock-in amplifier.

As is well known, the field modulation technique allows the suppression of time-dependent background signal drifts, such as those arising from a change in intensity of the microwave source or the gain of various components in the signal stream. Furthermore it facilitates lock-in detection where the system bandwidth (time constant) can be conveniently adjusted. A similar suppression can so be achieved with frequency modulation if the frequency dependence of the background is sufficiently flat; tuning to a maximum or minimum in the frequency-dependent transmission characteristics removes the background in first order. The FM approach is useful when studying resonances with a minimal field dependence (e.g., vortex resonances in magnetic nanostructures). With older klystron-based systems, which are subject to long-term drift, frequency modulation is often used to lock the source to a reference cavity; our HP83623A synthesizer is sufficiently stable that this is unnecessary.

Data acquisition was controlled and processed by various LABVIEW-based computer programs, which communicated with the microwave sweeper, magnet power supply, Gaussmeter, and lock-in amplifier through a general purpose interface bus. These features allow the system to be highly automated and also facilitates online signal processing (see below).

\section{TEST MEASUREMENTS}

To aid in the location of favorable frequencies to perform field sweeps in FMR measurements, the electromagnetic response of the system as a function of frequency was characterized whenever the probe was disassembled and reassembled. With the sample properly in place and the magnet off (producing only the residual dc field), a frequency sweep was performed. The center frequency and the range spanned can be controlled directly by the synthesizer. A slow sawtooth signal with an adjustable period, generated by the synthesizer and representing the instantaneous frequency, was fed to the computer. The signal, directly measured from the output of the microwave diode detector, was also fed into the computer as the transmitted signal amplitude. A schematic of this procedure is shown in Fig. 8.

Each configuration of the probe, consisting of the meanderline together with the sample, exhibits different electromagnetic transmission characteristics as a function of the frequency. ${ }^{16}$ Figure 9 illustrates a typical transmission amplitude versus frequency behavior $(10 \mathrm{MHz}-20 \mathrm{GHz})$ at zero magnetic field. The insets in Fig. 9 depict more detailed features of the spectrum as we zoom in on narrower frequency ranges. Rather than being broad band, the current devices display a high density of "transmission modes." However, we note that the device itself is unlikely to have flat transmission characteristics and in any case is not currently engi- 


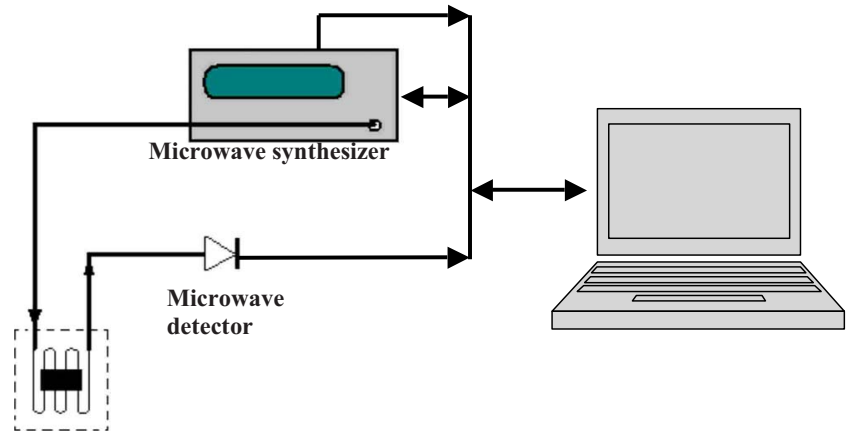

FIG. 8. (Color online) Electronics setup for electromagnetic transmission characterization.

neered to present a $50 \Omega$ impedance to the input transmission line. Hence one expects oscillations associated with standing waves on this line (which in our case is approximately $2.5 \mathrm{~m}$ in length); much of the oscillatory behavior should be suppressed by inserting a coaxial isolator (which was not available for this work).

The meanderline used here, which had lateral dimensions of $0.4 \times 0.4 \mathrm{in.}^{2}$ and a spacing of $0.013 \mathrm{in.} \mathrm{between}$ lines, was made from a 0.013 in. copper wire. The sample was a $0.19 \times 0.2 \mathrm{in}^{2}$ square array of $600 \mathrm{~nm}$ diameter, $25 \mathrm{~nm}$ thick Permalloy disks spaced by $1 \mu \mathrm{m}$ that was deposited and patterned on a silicon substrate. (FMR data involving this and other patterned samples will be presented elsewhere.) As can be seen in Fig. 9, there is a tendency for the intensity to decrease as the frequency is increased; at frequencies higher than $6 \mathrm{GHz}$ the intensity can drop by a few orders of magnitude relative to the lowest frequencies for our devices. A decrease in the intensity at high frequencies is expected due to various loss mechanisms: Ohmic heating, eddy-current loss to the surroundings, domain wall motion in the sample, etc.

A schematic of the electronic setup used for broad-band measurements is illustrated in Fig. 7. With the availability of the transmission characteristics obtained by the method de-

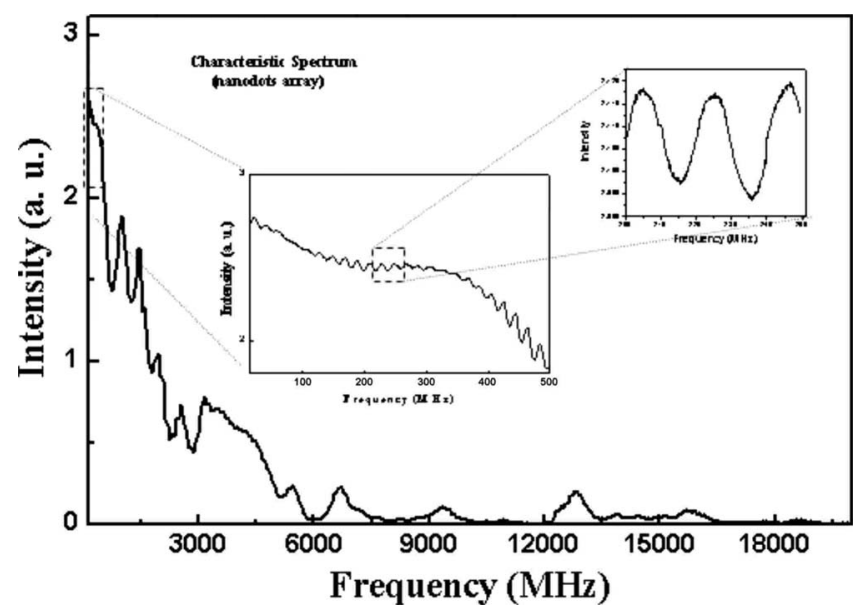

FIG. 9. A typical transmission spectrum for the system from $10 \mathrm{MHz}$ to $20 \mathrm{GHz}$. The meanderline has lateral dimensions of $0.4 \times 0.4 \mathrm{in}^{2}$ and is wound from a 0.013 in. copper wire; the sample is a $0.19 \times 0.2 \mathrm{in}^{2}$ Permalloy nanodot array on a $\mathrm{Si}$ substrate. The diagram in the middle inset is the spectrum in the range $10-500 \mathrm{MHz}$. The inset on the right shows spectrum in the range $200-250 \mathrm{MHz}$.

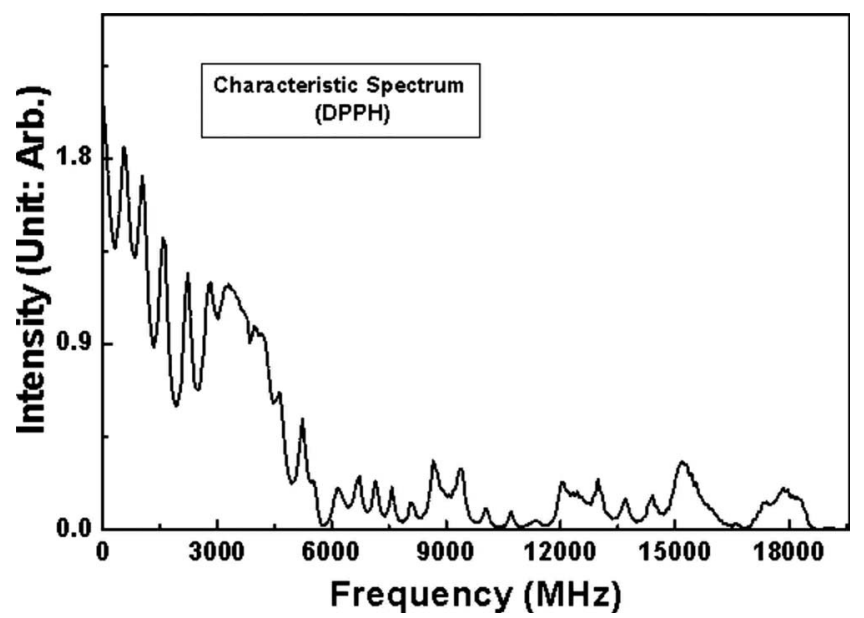

FIG. 10. Characteristic spectrum when DPPH is incorporated with the sample (the same meanderline in Fig. 9).

scribed, one might choose frequencies lying in windows where the transmission amplitude is locally maximal to perform magnetic field sweeps and record the spectrum. The criterion here is that the background should be minimal relative to a true signal when the field is swept at the selected frequencies. Typically the FMR uniform precession mode will be largest, but satellite resonances are commonly observed on patterned structures due to various spin-wave modes excited. However, there is no guarantee that the overall coupling to and from the spins is optimal at a transmission maximum and hence it is desirable to have an indicator internal to the probe, as we now discuss.

To better characterize the probe behavior when performing FMR measurements (where the internal alignment of the magnetization is inherently field-dependent) broadband measurements were made using 2,2-diphenyl-1-picrylhydrazyl (DPPH), a free radical material used in electron paramagnetic resonance (EPR) spectroscopy as a reference signal due to its high concentration of unpaired spins and precisely determined $g$ factor of 2.0036. For comparative studies, a sample was prepared by painting some varnish containing dissolved DPPH on the surface of a 0.1 $\times 0.2$ in $^{2} \mathrm{Si}$ substrate as a way to simulate a thin film sample. For reference we first characterized the transmission amplitude of the system for frequencies between $10 \mathrm{MHz}$ and $20 \mathrm{GHz}$ at zero fields, as shown in Fig. 10.

Absorption spectra were observed by performing magnetic field sweeps at numerous frequencies throughout the range of our microwave synthesizer. A typical DPPH absorption field-derivative curve at $17 \mathrm{GHz}$ is shown in Fig. 11(a). Using online software the data are numerically integrated to yield the undifferentiated resonance line(s). Figure 11(b) shows the integral of the data in Fig. 11(a). Also illustrated in the figure is a curve fit to a Lorentzian line shape. Here the fitted resonance field and the width were found to be 6191.3 and 18.965 Oe, respectively. Except for very low frequencies, DPPH resonances were observed throughout the frequency range of our spectrometer. Figure 12 illustrates some of the derivative absorption curves and the relative intensity at selective frequencies from 3 to $20 \mathrm{GHz}$. An example of the measured field/frequency dispersion relation for DPPH for a 

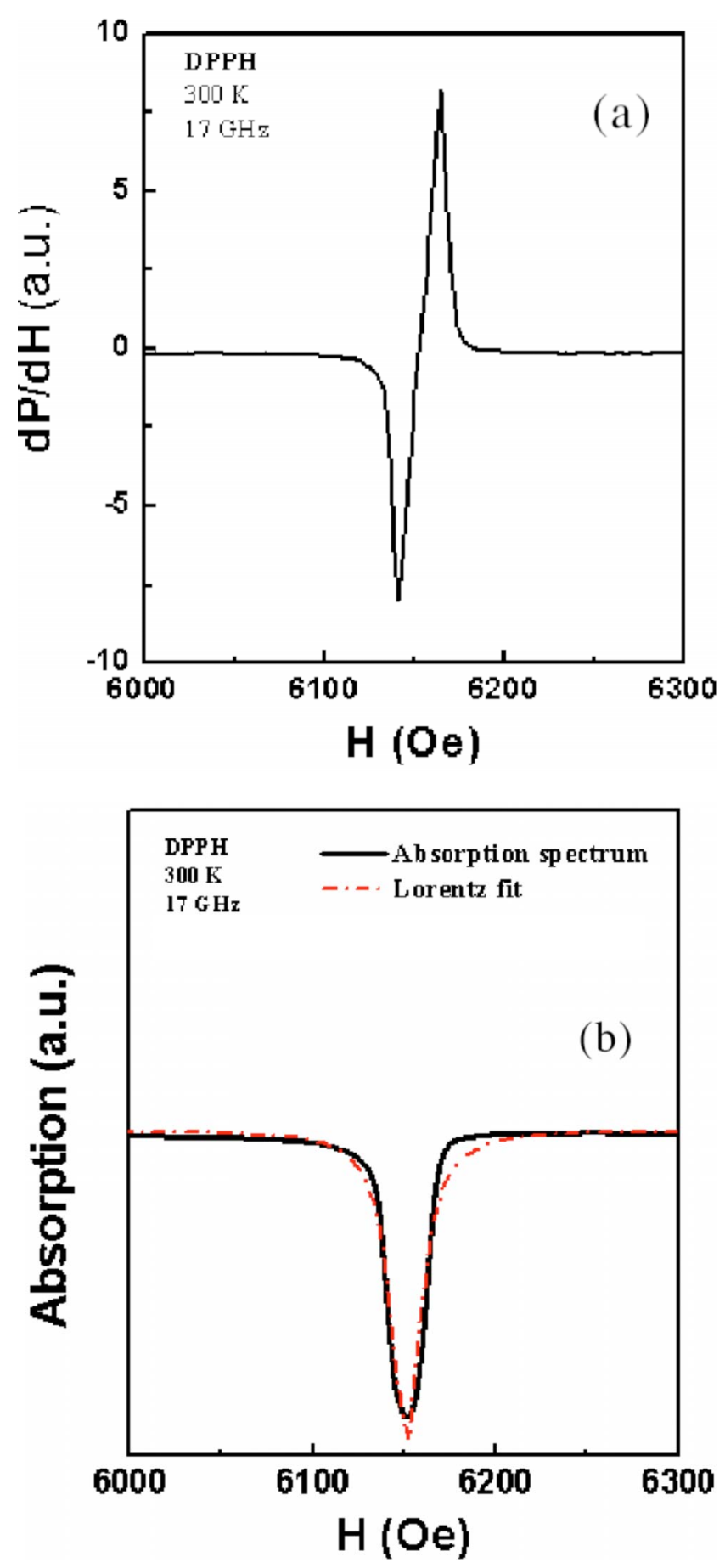

FIG. 11. (Color online) (a) Derivative of an absorption signal of DPPH at 17.0 GHz. (b) Resonance spectrum of DPPH at 17.0 GHz. The dashed line is a curve fit to a Lorentzian lineshape.

specific probe/sample setup is shown in Fig. 13. These data were taken with an uncalibrated Hall probe and gave a $g$ value of 1.966, which is smaller than the accepted 2.0035; this value was then used to calibrate the actual field produced by the magnet at the sample. (Factors such as a field differential between the Hall probe site and the sample site and any nonlinearities in the Hall probe can be compensated for in this way.) Comparison of the absorption intensity from the sample and the characteristic transmission response of the probe/sample system are illustrated in Fig. 14, which demonstrates that high absorption by the sample can occur at

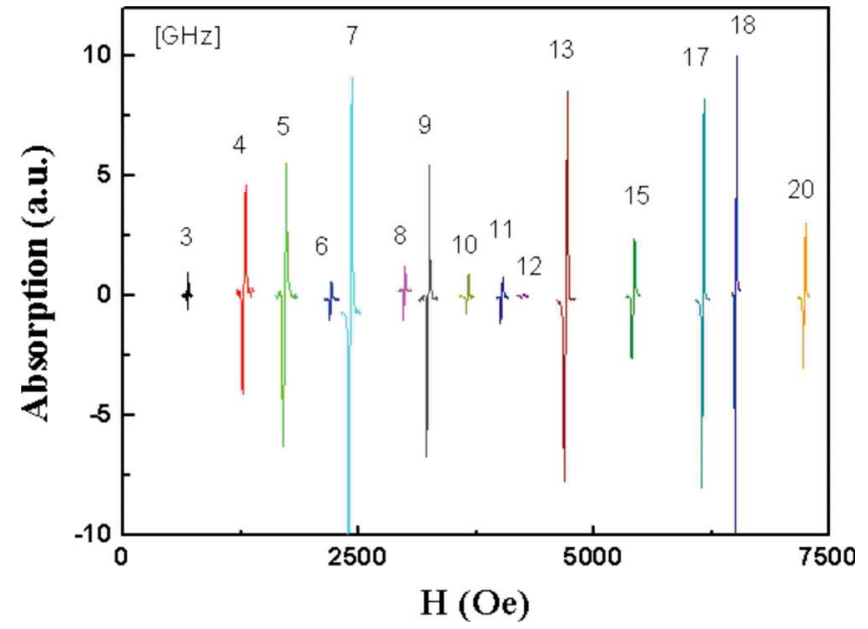

FIG. 12. (Color online) DPPH resonance derivatives at integral numbers of frequencies from 3 to $20 \mathrm{GHz}$.

frequencies where the transmission characteristic of the system was relatively low. Hence it is desirable to independently characterize the frequency dependence of both the electromagnetic transmission and the DPPH absorption intensity. Differences are presumably associated with resonances within the meanderline itself. Based on this observation, DPPH was routinely included along with the sample when performing FMR measurements and the resulting absorption then used as an index for choosing favorable frequencies for signal acquisition (FMR lines generally occur at widely differing fields from EPR lines minimizing any chance of confusion).

FMR spectra have been swept out using three modes of operation: (i) sweeping the magnetic field while modulating the field via the field coils, (ii) sweeping the magnetic field while frequency modulating the microwave source, and (iii) modulating the magnetic field while sweeping the microwave frequency. Mode (i) is the traditional approach, for which reason we will show no traces. Mode (ii) works quite well with our setup, particularly if a frequency is chosen

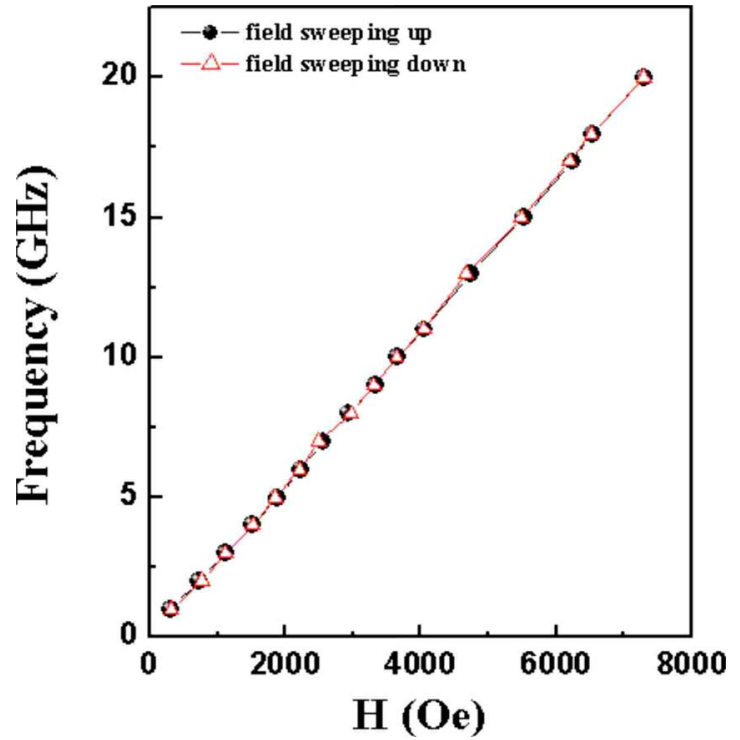

FIG. 13. (Color online) Measured frequency/field dispersion relation of DPPH. 

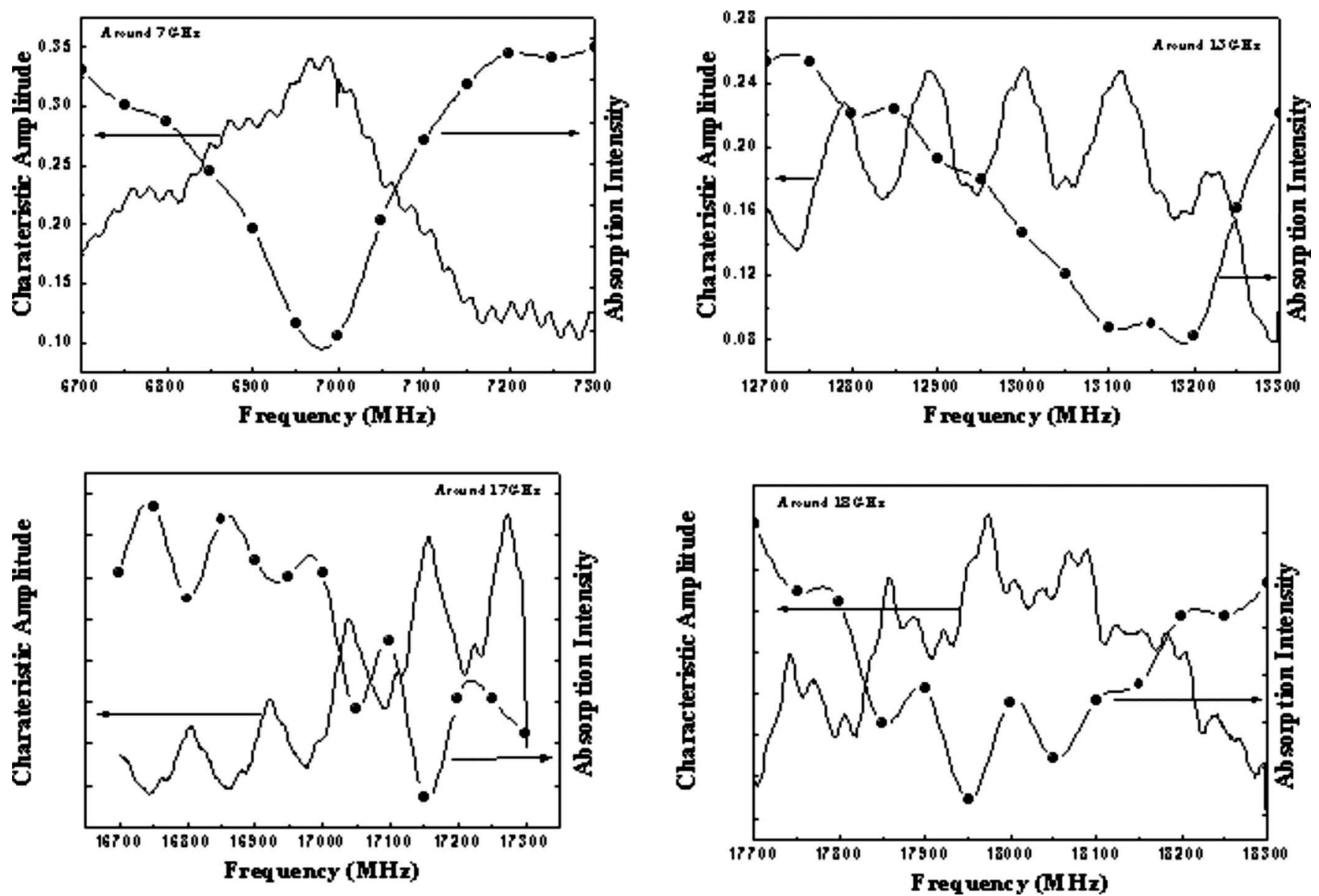

FIG. 14. Comparison of the absorption intensity from the sample and the characteristic transmission response of the system in the vicinity of the chosen frequencies of $7,13,17$, and $18 \mathrm{GHz}$. The curves associated with an arrow pointing to the right are for the absorption, while the characteristic transmission of the probe/sample system involves the curves with the arrow pointing to the left.

where the transmitted microwave signal is at a maximum or minimum where the AM accompanying the FM (which is what the lock-in detects) vanishes, thereby eliminating any offset. This mode can be useful in low temperature applications when eddy-current heating accompanying the ac field is present. Signals associated with mode (iii) will contain the envelope associated with the meanderline transmission characteristics. If the FMR line is narrow on this scale it will be

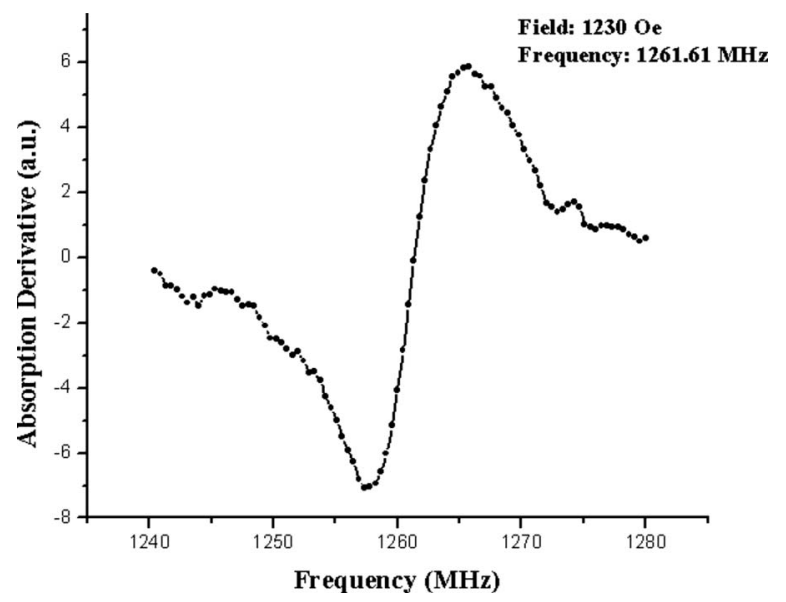

FIG. 15. FMR spectra of a $40 \mathrm{~nm}$ cobalt film obtained by sweeping the frequency at a fixed magnetic field of $1230 \mathrm{G}$ while modulating the field amplitude. minimally distorted. Figure 15 shows a spectrum collected on a $40 \mathrm{~nm}$ cobalt thin film taken in this mode; the position of the resonance is well defined although the wings are distorted for the reason mentioned. Mode (iii) allows data collection with a fixed magnetic field (a permanent magnet or a superconducting magnet in a persistent mode). A fourth combination involving sweeping the frequency while simultaneously frequency modulating it is not useful as the lock-in will primarily detect the frequency derivative of the meanderline transmission characteristic, which as discussed above has much structure.

To demonstrate the broad-band detection capability of our setup we show in Fig. 16 the resonance frequency as a function of magnetic field for the $40 \mathrm{~nm}$ cobalt film obtained primarily with mode (i) discussed above. Shown also is the prediction of the in-plane Kittel expression for the resonant frequency, $\omega=\gamma \sqrt{H\left(H+4 \pi M_{s}\right)}$. (The magnetization curves show only a small coercivity, and hence various anisotropy energy corrections that can enter the expression are suppressed.) The parameters used $(\gamma=2 \pi \times 3.2 \mathrm{GHz} / \mathrm{kOe}$ and $\left.4 \pi M_{s}=14.9 \mathrm{kG}\right)$ are taken from Ref. 17; the associated laboratory was the source from which the film was obtained.

\section{DISCUSSION AND CONCLUSIONS}

In this article, we have described a novel technique for performing broad-band FMR measurements on magnetically 


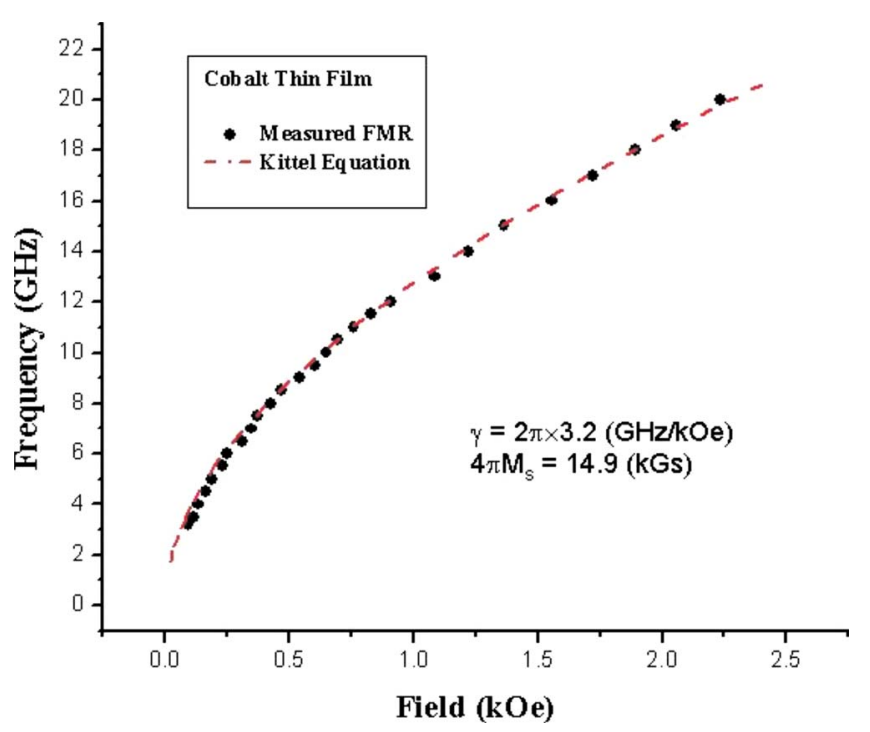

FIG. 16. (Color online) Field dependence of the FMR frequency of a $40 \mathrm{~nm}$ cobalt thin film. The solid line shows the predictions of the in-plane Kittel expression for the resonance frequency.

ordered thin film and patterned samples. Although the measurements discussed here are at room temperature, the approach has been applied at cryogenic temperatures. ${ }^{18} \mathrm{~A}$ key feature of our spectrometer is the use of a meanderline that closely couples to the sample and provides the required microwave "pumping" field. The frequency dependence of the amplitude of the transmitted signal through the overall system in the absence of a magnetic field shows a rather complex structure, including a number of resonances, both broad and sharp. In order to probe the overall response, including the dynamic coupling to a sample, the system was tested by measuring the response of DPPH where it was found that reasonably strong lines were observable over a, perhaps surprisingly, broad range of frequencies. As an example of a FMR application, data on a cobalt thin film were presented.

There is much room for further improvements. In particular, detailed modeling (simulations) of the electromagnetic response, including the coupling to and response of the sample, would surely improve the design of future probes, thereby yielding improved signal to noise. Inhomogeneities associated with the successive turns ("switchbacks") of the meanderline represent departures from a uniform transmission line and surely introduce a nonflat frequency response, the effect of which would emerge in detailed simulations. An additional effect is the interturn coupling, an effect associated with the exposed wires; this effect can be greatly suppressed by machining grooves in which the wire is embedded, with the sample covering the top of the grooves. Some preliminary experiments have been performed with such devices.

Another important research direction involves explicitly exploiting the periodicity associated with the line: a meanderline can be pictured as generating an excitation at a finite wavevector (and its spatial Fourier harmonics) which in turn can match finite wavevector excitations in the sample, e.g., bulk spin waves and surface Damon Eshbach modes in ferromagnets: ${ }^{19}$ this also includes diffusive modes such as spin diffusion in NMR. Although the associated meanderline wavelengths tend to be long relative to those of typical solid state magnetic excitations, the potential nonetheless exists to exploit this property. We note that the wires of our devices can be replaced by a patterned structure with much shorter spacings (on the micron scale); although the Ohmic losses would greatly increase, the metal could be replaced by a superconductor in low temperature applications. A particularly novel application would be the excitation of spin waves in superfluid and normal ${ }^{3} \mathrm{He} .{ }^{20}$

Finally, it would be interesting to use meanderlines to study various nonlinear effects in FMR associated with large tipping angles of the magnetization. Such effects have a long history involving harmonics and subharmonics of the excitation field. ${ }^{21}$ Meanderlines might also be used to study sum and difference frequency generations.

\section{ACKNOWLEDGMENTS}

This research was supported by the National Science Foundation under Grant Nos. DMR-0509357 and DMR0509691 and by the Korea Science and Engineering Foundation (KOSEF) through the National Research Laboratory funded by the Ministry of Science and Technology (Grant No. ROA-2006-000-10241-0).

${ }^{1}$ S. Jung, B. Watkins, L. De Long, J. B. Ketterson, and V. Chandrasekhar, Phys. Rev. B 66, 132401 (2002).

${ }^{2}$ V. Novosad, F. Y. Fradin, P. E. Roy, K. Buchanan, K. Yu. Guslienko, and S. D. Bader, Phys. Rev. B 72, 024455 (2005).

${ }^{3}$ K. Rivkin, W. Xu, L. E. De Long, V. V. Metlushko, B. Ilic, and J. B. Ketterson, J. Magn. Magn. Mater. 309, 317 (2007).

${ }^{4}$ G. N. Kakazei, P. E. Wigen, K. Yu. Guslienko, V. Novosad, and A. N. Slavin, Appl. Phys. Lett. 85, 443 (2004).

${ }^{5}$ Y. Tsuchiya, K. Takai, N. Momo, T. Nagami, H. Mizuta, S. Oda, S. Yamaguchi, and T. Shimada, J. Appl. Phys. 100, 094306 (2006).

${ }^{6}$ R. P. Cowburn, J. Magn. Magn. Mater. 242, 505 (2002).

${ }^{7}$ C. Tsang, R. E. Fontana, T. Lin, D. E. Heim, V. S. Speriosu, B. A. Gurney, and M. L. Williams, IEEE Trans. Magn. 30, 3801 (1994).

${ }^{8}$ M. Scheffler, G. Tröster, J. L. Contrras, J. Hartung, and M. Menard, Microelectron. J. 17, 11 (2000).

${ }^{9}$ D. R. Young, J. C. Swihart, S. Tansal, and N. H. Meyers, Solid-State Electron. 1, 378 (1960).

${ }^{10}$ W. H. Henkels and C. J. Kircher, IEEE Trans. Magn. 13, 63 (1977).

${ }^{11}$ B. W. Langley, S. M. Anlage, R. F. W. Pease, and M. R. Beasley, Rev. Sci. Instrum. 62, 1801 (1991).

${ }^{12}$ V. P. Denysenkov and A. M. Grishin, Rev. Sci. Instrum. 74, 3400 (2003).

${ }^{13}$ V. Castel, J. B. Youssef, and C. Brosseau, J. Nanomater., Special Issue 1, Article ID27437, 2007 (2007).

${ }^{14}$ J. B. Youssef and C. Brosseau, Phys. Rev. B 74, 214413 (2006).

${ }^{15}$ Y. Guan and W. E. Bailey, Rev. Sci. Instrum. 77, 053905 (2006).

${ }^{16} \mathrm{We}$ are unaware of any calculations for the transmission characteristics or field distribution of our wire-wound meanderline next to a conducting substrate. The case of a straight wire or strip adjacent to a perfectly conducting ground plane is treated in Secs. 5.7.4 and 5.7.5 in D. Sengupta and V. Liepa, Applied Electromagnetics and Electromagnetic Compatibility (Wiley, New York, 2006).

${ }^{17}$ J. G. Kim, K. H. Han, J. H. Cho, and S. Lee, Phys. Status Solidi B 241, 1721 (2004).

${ }^{18}$ I. P. Nevirkovets, O. Chernyashevskyy, J. B. Ketterson, V. Metlushko, and B. K. Sarma, J. Appl. Phys. 104, 063920 (2008)..

${ }^{19}$ S. A. Bogacz and J. B. Ketterson, J. Appl. Phys. 58, 1935 (1985).

${ }^{20}$ S. A. Bogacz and J. B. Ketterson, Phys. Rev. Lett. 57, 591 (1986).

${ }^{21}$ For an early review see Ferromagnetic Resonance at High Power, R. W. Damon, in Magnetism, edited by G. Rado and H. Suhl, (Academic, New York, (2004), Vol. I. 